\title{
Local Signals From Beyond the Receptive Fields of Striate Cortical Neurons
}

\author{
James R. Müller, ${ }^{1}$ Andrew B. Metha, ${ }^{1}$ John Krauskopf, ${ }^{2}$ and Peter Lennie ${ }^{1}$ \\ ${ }^{1}$ Center for Visual Science and Department of Brain and Cognitive Sciences, University of Rochester, Rochester, 14623; \\ ${ }^{2}$ Center for Neural Science, New York University, New York City, New York 10003
}

Submitted 6 January 2003; accepted in final form 17 April 2003

\begin{abstract}
Müller, James R., Andrew B. Metha, John Krauskopf, and Peter Lennie. Local signals from beyond the receptive fields of striate cortical neurons. J Neurophysiol 90: 822-831, 2003. First published April 30, 2003; 10.1152/jn.00005.2003. We examined in anesthetized macaque how the responses of a striate cortical neuron to patterns inside the receptive field were altered by surrounding patterns outside it. The changes in a neuron's response brought about by a surround are immediate and transient: they arise with the same latency as the response to a stimulus within the receptive field (this argues for a source locally in striate cortex) and become less effective as soon as $27 \mathrm{~ms}$ later. Surround signals appeared to exert their influence through divisive interaction (normalization) with those arising in the receptive field. Surrounding patterns presented at orientations slightly oblique to the preferred orientation consistently deformed orientation tuning curves of complex (but not simple) cells, repelling the preferred orientation but without decreasing the discriminability of the preferred grating and ones at slightly oblique orientations. By reducing responsivity and changing the tuning of complex cells locally in stimulus space, surrounding patterns reduce the correlations among responses of neurons to a particular stimulus, thus reducing the redundancy of image representation.
\end{abstract}

\section{IN T R O D U C T I O N}

"End-stopping" and "side-stopping" are well-known properties of receptive fields in striate cortex (V1) (Hubel and Wiesel 1965; Maffei and Fiorentini 1976). A pattern that extends beyond the receptive field often elicits a weaker response than a pattern that just fills it. This surrounding zone of inhibition is not generally considered part of the receptive field because stimuli falling on it cannot by themselves elicit a response. The most suppressive surrounding patterns usually have the same orientation and spatial frequency as those to which the receptive field is best tuned (Blakemore and Tobin 1972; Cavanaugh et al. 2002b; DeAngelis et al. 1994; Gilbert and Wiesel 1990; Levitt and Lund 1997; Nelson and Frost 1978; Sillito et al. 1995).

It has been argued that the surround helps figure-ground segregation by suppressing signals within patches of uniform texture (Bradley and Andersen 1998; Lamme 1995; Sillito et al. 1995; Tanaka et al. 1986) and that it adjusts the responsivity of a neuron to the ambient contrast in the neighborhood of the receptive field (Cavanaugh et al. 2002a). We wondered if the surround might confer an additional benefit. In natural images, the correlation between the statistics of two regions declines with the separation of the regions (Simoncelli and Schwartz

Address for reprint requests: J. R Müller, Howard Hughes Medical Institute and Dept. of Neurobiology, Fairchild D209, Stanford University School of Medicine, Stanford, CA 94305-5125 (E-mail: jim@monkeybiz.stanford.edu).
1999). If a pattern falling on the surround consistently suppressed a neuron's response to a pattern of similar orientation falling on the receptive field, it might repel the neuron's orientation tuning curve, thereby reducing the correlation (redundancy) among visual signals that arise from adjacent image regions of similar structure. In this respect, the surround would act in the spatial domain in much the same way that rapid contrast adaptation (Müller et al. 1999) acts in the time domain.

In this paper, we explore the mechanism of surround suppression to characterize its latency and persistence and to ask whether it acts by subtraction or division. We describe how reductions in response reduce redundancy of image representation among neurons with neighboring or overlapping receptive fields: specifically, we show that surround suppression changes orientation selectivity and that these orientation-specific changes in sensitivity reduce redundancy.

\section{METHODS}

Action potentials were recorded from single neurons in striate cortex of 14 anesthetized Macaca fascicularis. Preparation and general methods were as described in Müller et al. (2001).

\section{Visual stimuli}

Sinusoidal gratings were generated as described in Müller et al. (2001). Displays often required two gratings whose spatial and temporal characteristics could be controlled independently. These were produced on a single monitor by dividing the 256 available lookup table entries into two independent sets of 128 entries, one allocated to each pattern. The display was viewed from a distance of $114-342 \mathrm{~cm}$, depending on the resolving power of the neuron under study, and its height subtended $11.1-3.8^{\circ}$. It was refreshed at $75 \mathrm{~Hz}$, with the scan from top to bottom of the screen taking $\sim 10 \mathrm{~ms}$.

\section{Characterizing receptive fields}

The basic characteristics of receptive fields, including their positions and dimensions, were established as described in Müller et al. (2001). Briefly, receptive fields were first mapped using a small patch of moving grating whose spatial and temporal characteristics were continuously adjustable by the experimenter. The preferred position, size, orientation, and spatial frequency derived from this examination were then used as the starting points for systematic measurement of each of these characteristics. The position of the receptive field was established using a patch of moving grating, of length and width approximately matching the receptive field, presented at a matrix of

\footnotetext{
The costs of publication of this article were defrayed in part by the payment of page charges. The article must therefore be hereby marked "advertisement" in accordance with 18 U.S.C. Section 1734 solely to indicate this fact.
} 
positions centered on the estimated position and spaced 0.25 lengths and widths apart. Having found the receptive field position, we then established the rectangle (lying in the preferred orientation of the neuron) that best matched the receptive field in size. To establish the optimal length, we used a series of gratings of different lengths with width fixed at a preliminary estimate; to establish the preferred width, we used a series of gratings of different widths with length fixed at the length preferred. We then established the optimal spatial phase using flashed gratings at a series of phases. If at any stage in this sequence of measurements it appeared that some estimate was incorrect, we repeated the sequence. To characterize the influence of the region surrounding the receptive field, we presented a grating of optimal size, of the highest contrast that did not saturate the response, together with a surrounding grating that enclosed but did not overlap the rectangle that bounded the receptive field and was also of high contrast. The outer boundary of this surround usually extended to $2^{\circ}$ from the center of the receptive field - a region that preliminary observations showed to be large enough to capture essentially all of the surround's influence (see also Cavanaugh et al. 2002a; Levitt and Lund 2002). In control experiments, we established that a surround pattern presented alone elicited no excitatory response from a neuron. If the control failed, the inner boundary of the surround (chosen initially to abut the estimated receptive field) was enlarged in stages, leaving a gap between the surround and any pattern placed on the receptive field. Occasionally the surrounding pattern presented alone slightly depressed the neuron's spontaneous discharge. In such cases, we did not enlarge the surrounding pattern to leave a gap. We studied neurons' responses to moving gratings and to stationary gratings in optimal spatial phase. Respiration-induced eye movements (Forte et al. 2002) were occasionally large enough to prevent satisfactory measurement of the responses of simple cells to stationary gratings of high spatial frequency.

\section{Characterizing orientation selectivity}

For parts of our analysis, we needed to characterize a change in the shape of an orientation tuning curve, generally a deformation that shifted the peak of the tuning curve and introduced an asymmetry. To capture this, we calculated the center-of-mass of the tuning curve $\sum i R_{i} / \sum R_{i}$ where the $R_{\mathrm{i}}$ denote the responses to gratings in a range of orientations $i$, either $i \in\left\{0, \pm 7, \pm 14, \pm 21\right.$, and $\left.\pm 28^{\circ}\right\}$ or $i \in\{0$, $\pm 18, \pm 36, \pm 54, \pm 72$, and $\left.\pm 90^{\circ}\right\}$ where $0^{\circ}$ is the nominal preferred orientation. For a neuron with a symmetrical, unimodal tuning curve that is well sampled by the test orientations the center-of-mass is simply the preferred orientation.

\section{RES U L T S}

Our analysis is based on recordings from 58 well-characterized neurons in V1 (15 simple cells, 43 complex cells). All receptive fields lay within $3^{\circ}$ of the center of the fovea. Not all experiments were run on all cells encountered. The proportions of simple and complex cells do not reflect rates of encounter: for some experiments we sought complex cells only.

\section{Mechanism}

Because the surround's influence is expressed only by modulating responses to patterns falling on the receptive field, we infer it from the change the surrounding pattern brings about in the response to an optimal grating on the receptive field. In principle, signals from outside the receptive field might originate from one neuron or from many-in the lateral geniculate nucleus (LGN), within V1, or in extrastriate cortex - and might act subtractively or divisively. A detailed understanding of the time course of surround effects would help us to approach these questions.

\section{Rapid, transient influence}

Because neurons of the type commonly encountered in cortex respond transiently to a stationary stimulus (Müller et al. 2001), we would expect the influence of a stationary surround to be transient. Figure 1 shows, for three neurons, the average discharge rate during the first $100 \mathrm{~ms}$ of response to the presentation of an optimal stationary grating in the receptive
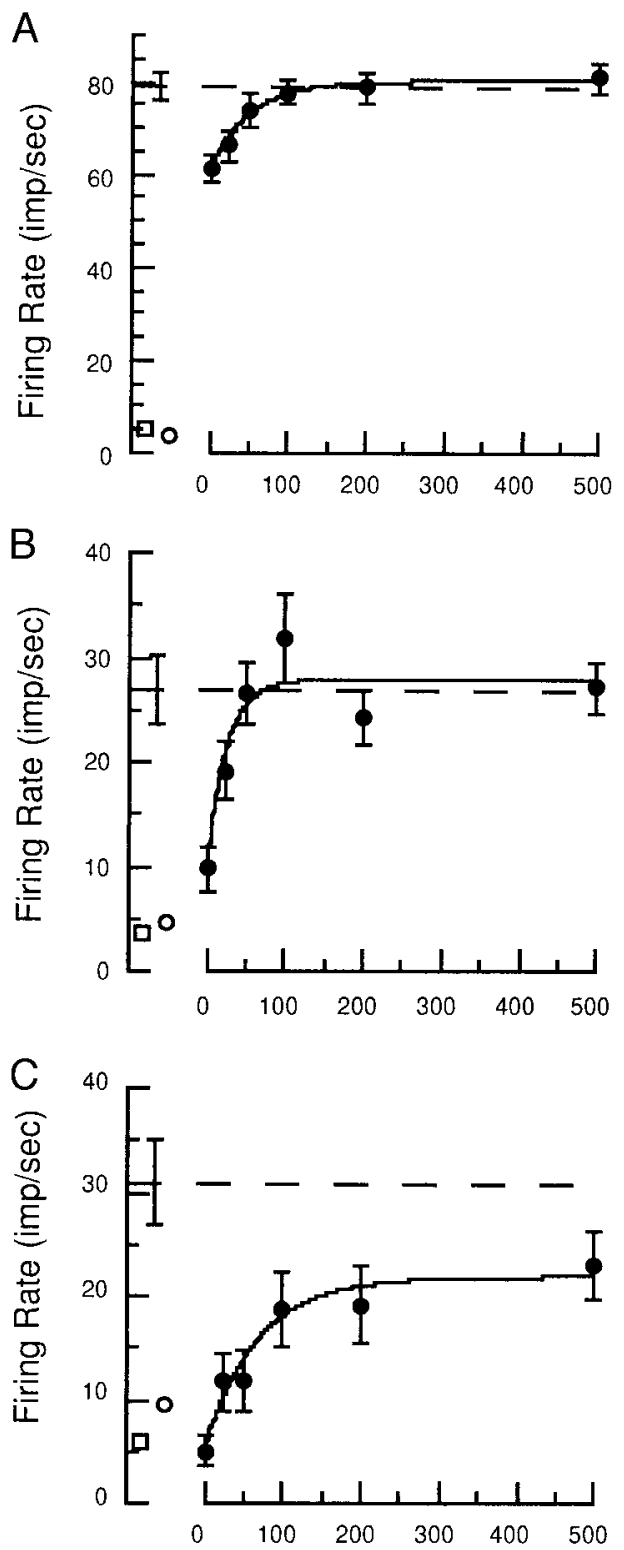

\section{Onset Time of Optimal Grating re Onset Time of Surround (msec)}

FIG. 1. Time course of the influence of a surrounding grating on the response to a grating confined to the receptive field. Both gratings were stationary and of optimal orientation and spatial frequency. Each panel shows a different complex cell. Discharge rates are averages over the $1 \mathrm{st} 100 \mathrm{~ms}$ of a neuron's response, which always followed the presentation of the receptive field grating with a fixed latency. - - -, the neuron's response to the optimal grating presented alone. Points at the bottom left of each graph show discharge in the absence of any grating ( $\square$ ), and in the presence of the surrounding grating alone $(\mathrm{O})$. $\bullet$, the responses to the optimal grating delivered at different times after the onset of the surrounding one. Error bars show \pm 1 SE. Smooth curves show best-fitting solutions to $E q$. 1 . 
field as a function of the time after surround onset at which this optimal grating was presented. The graphs therefore trace out the time course of the surround's transient influence. The response is most suppressed when center and surround gratings are presented synchronously and recovers rapidly-in two cases completely - as the interval between the onsets of the surround grating and the central grating approaches $100 \mathrm{~ms}(A$ and $B$ ). For asynchronies greater than that bringing about the weakest response $R_{\min }$, the discharge rate $R$ is well described by an exponential recovery (Fig. $1,-$ )

$$
R=\left(R_{\min }-R_{\text {ayympt }}\right) e^{-t / \tau}+R_{\text {asympt }}
$$

where $R_{\text {asympt }}$ is the response when asynchrony is large, $t$ is the surround onset asynchrony relative to that which is most suppressive, and $\tau$ is the time constant of recovery. If $R_{\mathrm{rf}}$ is defined as the response to the optimal receptive field stimulus alone, the recovering responsivity can be conveniently characterized by its time constant $\tau$ and by the ratio $\left(R_{\text {asympt }}-R_{\mathrm{rf}}\right) /\left(R_{\min }-\right.$ $R_{\mathrm{rf}}$ ), which represents the extent to which the surround's influence is sustained.

The measurements and analysis illustrated in Fig. 1 were made on 16 neurons. Figure 2 summarizes them. Surround effects decay quickly, often to small values (negative values mean that the asymptotic discharge rate in the presence of a surrounding grating was higher than when the receptive field alone was stimulated). The distribution of time constants is like that for the decay of responses to stationary gratings confined to the receptive field (Müller et al. 2001) (Fig. 2), but the sustained surround effects have a broader distribution of amplitudes.

For most neurons (12/16) on which we examined the effects of gratings presented asynchronously to the surround and receptive field, the surround exerted its greatest influence when it appeared simultaneously with the grating on the receptive field (e.g., Fig. 1). This implies that surround signals act on a neuron as rapidly as do those arriving through the receptive field. We explored this further by looking at the fine structure of responses to gratings falling on receptive field and surround.

We analyzed cumulative spike counts of the kind shown in Fig. 3. Figure $3 A$ shows, for a complex cell that had no spontaneous activity, the cumulative count following the presentation of an optimal grating on the receptive field, alone (-) and concurrently with a surrounding grating of the same orientation (- -). The surrounding grating completely extinguished the response. The progressive scan of the monitor ensures that parts of the surrounding grating are displayed before the stimulus to the receptive field, and parts are delivered later. Assuming that the dominant surround signals originate $0.5^{\circ}$ beyond the receptive field (Born and Tootell 1991; Cavanaugh et al. 2002a; Levitt and Lund 2002; Maffei and Fiorentini 1976) and that all of these signals arise in the part stimulated earliest, we estimate that the surround might be stimulated as much as $1.25 \mathrm{~ms}$ earlier than the receptive field. Taking this as the precision of our measurement, surround signals act as quickly as those arising through the receptive field. Figure $3 B$ shows a corresponding set of traces obtained with an orthogonal surrounding grating that increased the neuron's responsivity. The discharge departs from baseline sooner when the surrounding grating is present. This might mean that surround signals are available before signals that arrive through the receptive field first drive a neuron's mem-
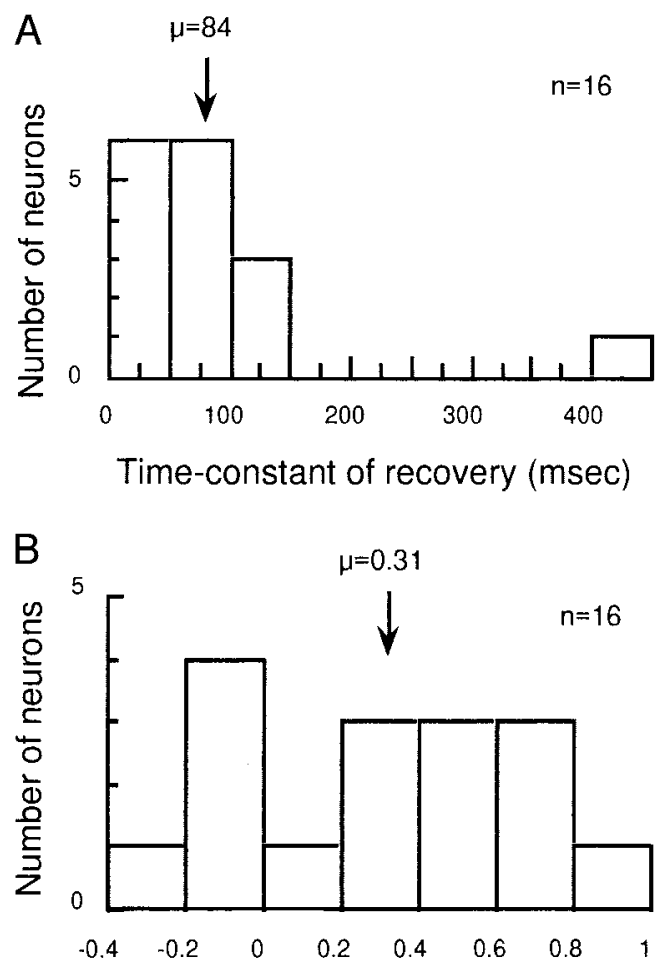

Relative Amplitude of Sustained Effect

FIG. 2. Temporal characteristics of surround influence studied with stationary gratings delivered to the receptive field and to the surround. $A$ : distribution of time constants of the recovery of responsivity following surround onset. $B$ : distribution of the extent to which the effect of the surround is sustained, the ratio $\left(R_{\text {asympt }}-R_{\mathrm{rf}}\right) /\left(R_{\min }-R_{\mathrm{rf}}\right)$.

brane potential to its spike threshold. To explore that issue, we looked at the time course of the suppression brought about by a surround presented after the onset of the grating falling on the receptive field. This is shown, for another cell, in Fig. $3 C$. Measured in this way the suppressive influence of the surround had a latency of $32 \mathrm{~ms}$ - faster than the response to a grating confined to the receptive field of this neuron $(42 \mathrm{~ms})$ or any other (see following text, Fig. 4B).

Measurements of the kind shown in Fig. 3 were made on 37 neurons and are summarized in Fig. 4. Figure $4 A$ shows the average cumulative spike counts obtained from 37 neurons following the onset of a grating that just filled the receptive field (-) or covered both the receptive field and the surround (- - -). Before averaging, the traces for individual neurons were aligned to the time of onset of the response to the optimal grating. The points at which the slopes of the stimulus-driven traces begin to differ from the slope of the trace of the maintained discharge $(\cdots)$ and from each other show the latencies of the signals arising from the optimal grating and the pattern surrounding it respectively. Both signals begin abruptly and influence discharge at the same time.

Figure $4 B$ shows more detail about the behavior of individual neurons. For each neuron, the absolute latency of response to an optimal grating that filled the receptive field is plotted against the absolute latency of the influence of a surrounding grating of matching orientation, measured by the method shown in Fig. 3. Most neurons receive surround signals and receptive field signals at the same time. For a few (such as 
A

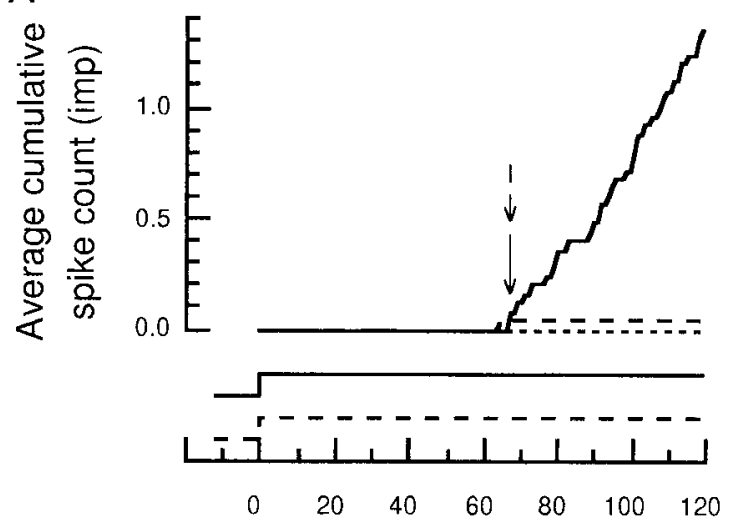

B
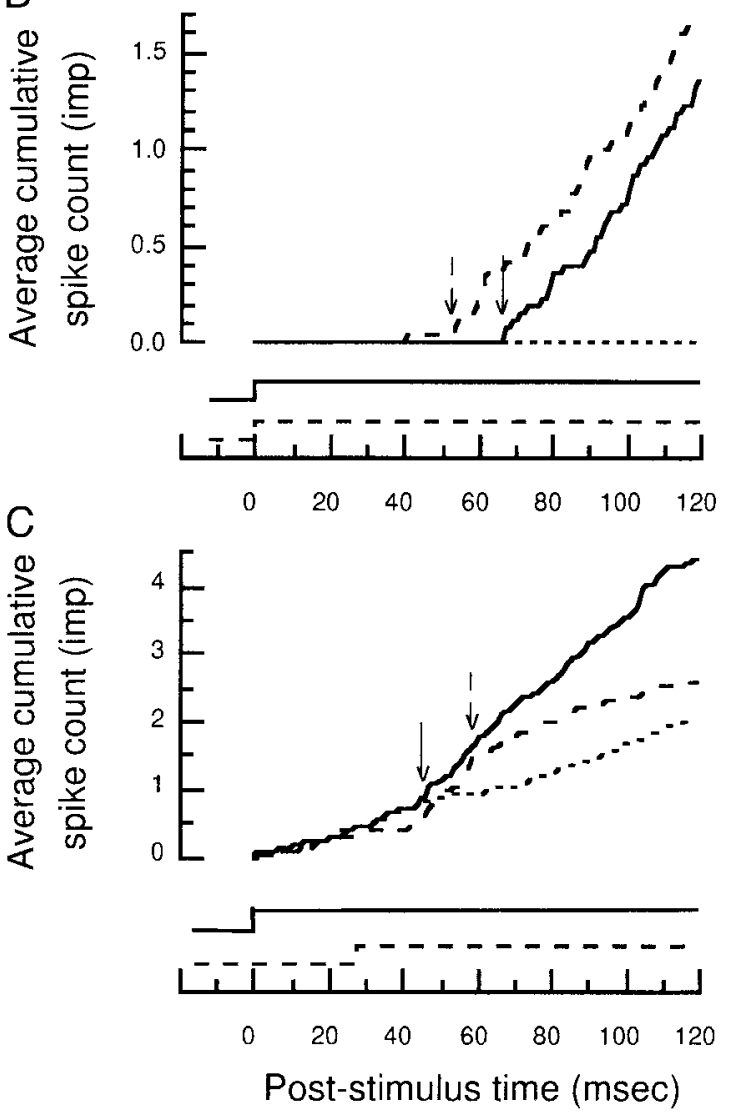

FIG. 3. Illustration of method for characterizing latency of response. $A$ : cumulative spike counts after the onset of an optimal grating on the receptive field, alone (-) and when presented concurrently with a surrounding grating (--) of the neuron's preferred orientation. The cell had no spontaneous activity (- - ). Traces below show the time of onset of the central (-) and surrounding (- - ) grating. Spike times measured to the nearest $100 \mu \mathrm{s}$. Points at which the stimulus-driven traces diverge from the trace of the maintained discharge and from each other (solid and dotted arrows, assessed by eye) are taken as the latencies of the signals arising from the optimal grating and the pattern surrounding it respectively. $B$ : same as $A$ except surrounding grating in the orthogonal orientation. $C$ : same as $A$ (for another neuron) except surrounding grating presented $27 \mathrm{~ms}$ after the onset of the grating on the receptive field.

that of Fig. $3 C$ ) the surround is faster; for a few others it is slower.

The more reliable the effect of the surround, the more likely it is to exert its influence at the moment the response begins.
Figure 5, shows how the latencies of surround signals (expressed relative to the latency of signals originating within the receptive field) vary with the strength of the surround's influence, expressed in $d^{\prime}$ units (Eq. 2, measured using the 1 st 100 $\mathrm{ms}$ of discharge). Figure $5 A$ shows the latencies when the surrounding grating lay at the orientation optimal for the receptive field; Fig. $5 B$ shows latencies when the surrounding grating lay at the orientation orthogonal to the optimal grating on the receptive field. For most cells, a surrounding grating, whether suppressing or facilitating the response, acts just as rapidly as a grating confined to the receptive field. Only for those cells showing the least reliable surround influence was that influence delayed by $\geq 10 \mathrm{~ms}$; similarly, some of cells showing the most advanced surround influence were among the least reliable.

\section{Divisive gain control}

Our use of stationary gratings to stimulate the receptive field provides an opportunity to establish whether surround signals act through division or subtraction. A stationary grating presented to the receptive field brings about a brisk response that declines over the course of a few hundred milliseconds. To characterize the nature of the surround's effect (subtractive/ divisive), we presented the surrounding grating, on separate trials, at a range of times shortly after presenting a stationary grating to the receptive field. If the surround acts subtractively, the presentation of a surrounding pattern at any time after the onset of the pattern on the receptive field will lead to a constant reduction in response. On the other hand, if the surround acts divisively, the effect of its presentation will shrink with the decaying response to the stationary pattern on the receptive field. The filled symbols in Fig. $6 A$ show, for one neuron, the response after the onset of a stationary grating confined to the receptive field, sampled in 100-ms epochs, each beginning at the poststimulus time indicated by the symbols (some of these epochs overlap). Open symbols show the response to the same grating, sampled in the same way, but now from a different set of trials in each of which a surrounding grating was presented just before one of the sampling epochs (by a fixed interval equal to the neuron's onset latency), so that the added grating begins to influence discharge immediately before the beginning of the epoch indicated by the symbol. In other words, the open symbols show what would be the response to the presentation of a stationary grating in the receptive field, in the presence of a surround of constant potency. The dotted line shows the uppermost curve displaced so as to best fit the response obtained in the presence of the surrounding grating. This is what one might expect were the surround acting subtractively, but it does not characterize the data well.

The response in the presence of the surrounding grating is much better described by assuming that a newly presented surrounding grating has the same divisive effect at any time during the response to the grating in the receptive field. This is shown by the dashed line, which is a reduced-scale replica of the uppermost curve, fitted after discounting the maintained discharge.

We made measurements of this kind on $n=8$ neurons and fit subtractive and divisive models as in the preceding text. Fit quality was measured by the percentage of variance in the data that each model left unaccounted for (Fig. 6B): the model's 

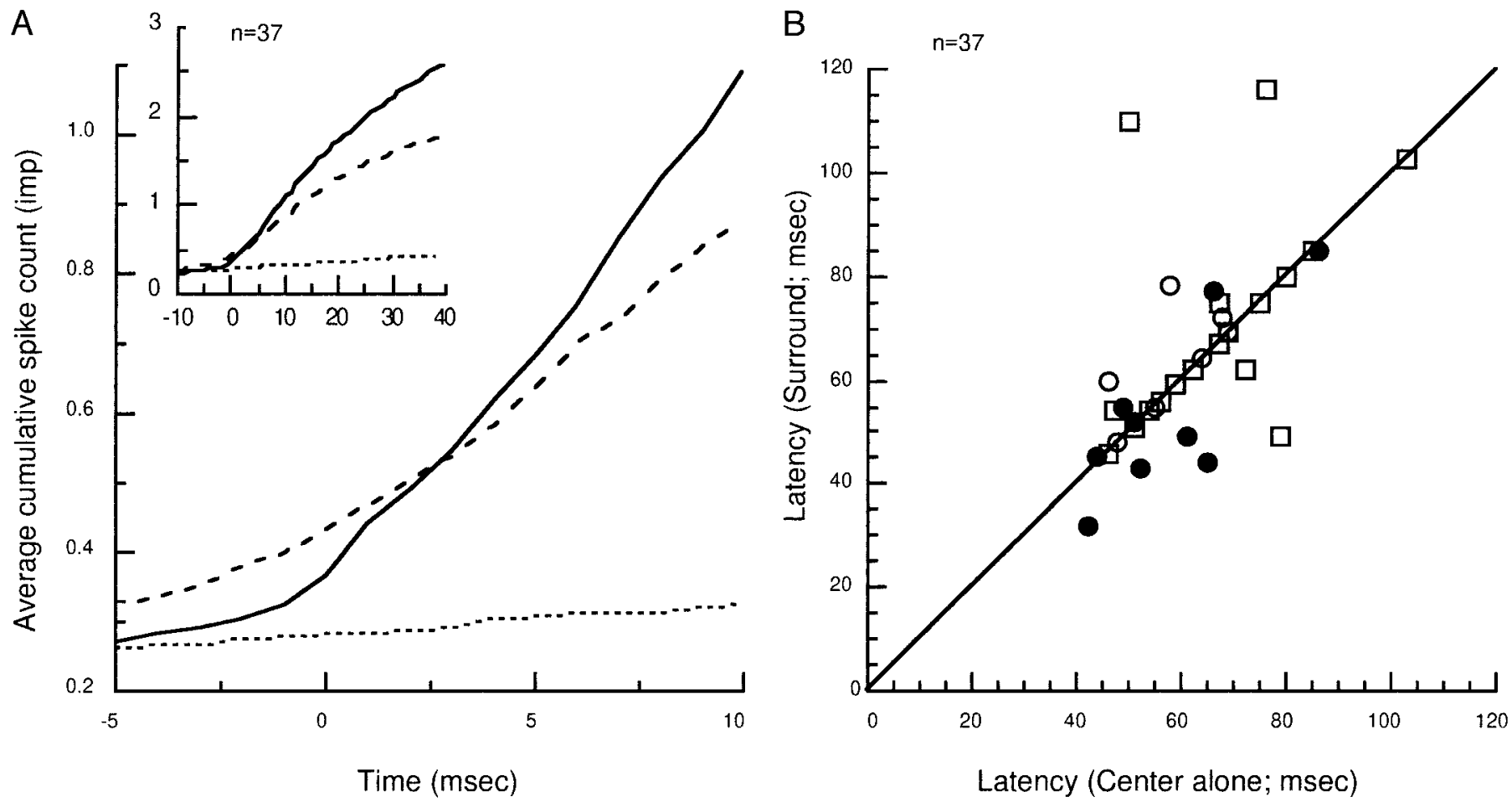

FIG. 4. Latencies of signals arising in the receptive field and in the surround, for a population of 37 neurons. A: average growth of discharge in response to an optimal grating filling each neuron's receptive field (-) and to a grating of the same orientation that covered both the receptive field and the surround (- -) and when no stimulus is presented $(\cdots)$. Traces show cumulative spike counts after stimulus onset averaged after aligning individual responses to the time when each neuron first responded to its optimal grating. Inset: the same cumulative average counts, extending over a longer period of time. $B$ : absolute latencies of individual neurons measured by the method shown in Fig. 3. Measurements were made with a stationary grating of optimal orientation and phase delivered to the receptive field and a similar grating delivered to the surround concurrently ( $\square$ ) or delayed by $27 \mathrm{~ms}(\bullet)$; measurements were also made with moving gratings whose spatial phase at onset was one to which the neuron responded well $(0)$. Same neurons as in $A$.

mean-squared fit error divided by the mean-squared error of the best-fit horizontal line. This quantity is zero for perfect fits, 100 for fits that are just as good as the best horizontal line, and potentially larger when data are noisy. We used a permutation test (Edgington 1995) to determine whether a subtractive or a divisive surround better explained the results. The null hypothesis was that the distributions of fit quality for the two models were the same (each model has exactly 1 free parameter). A simulated data set was drawn randomly from that distribution by choosing each of the $2 n$ actual fit qualities without replace-
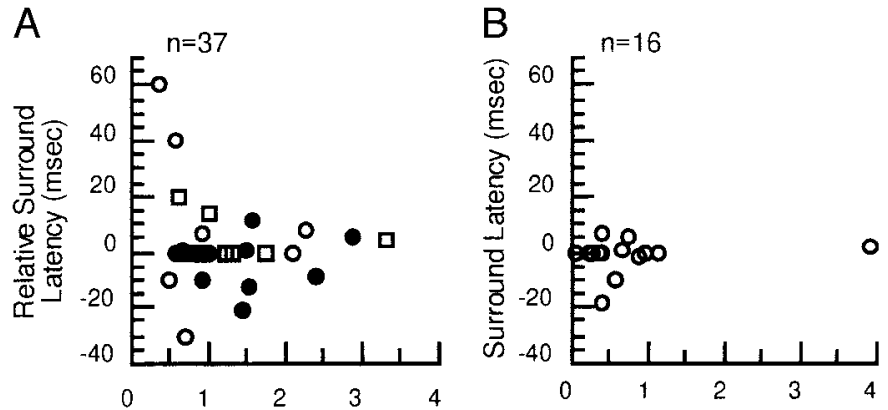

Size of Surround Effect ( $d$ ' units)

Size of Surround Effect (d' units)

FIG. 5. A: latency of surround signals in relation to latency of signals arising in the receptive field, plotted against the strength of surround influence, characterized by $d^{\prime}$ (see Eq. 2) Same neurons as in Fig. 4. Same symbols as in Fig. $4 B$. $B$ : same as $A$ except the surround grating was orthogonal to the optimal grating in the receptive field. ment. This was repeated 5000 times. To assess statistical significance we computed a two-tailed $P$ value, the probability that a mean absolute difference between distributions of fit qualities for the two models as simulated was greater than or equal to that for the actual fits. Division explained significantly more of the variance than subtraction $(P<0.01$, permutation test). The divisive model left $8 \%$ of the variance unaccounted for in the median case; the subtractive model left $21 \%$. For seven of these neurons the divisive model fit well, leaving $<15 \%$ of the variance unaccounted for (Fig. $6 B$ ).

\section{Influence on orientation selectivity}

To establish in detail how the presence of a surrounding grating influences a neuron's orientation tuning, we measured responses to a set of optimally sized gratings of the preferred spatial frequency at a range of orientations, alone, and in the presence of each of two surrounding gratings that lay in different orientations slightly oblique to the preferred orientation.

We made measurements with stationary gratings in optimal phase as well as with moving gratings. When using stationary gratings we measured the first $100 \mathrm{~ms}$ of response, which captures the onset transient (Müller et al. 2001). When using moving gratings, we also analyzed the onset transient (initial $100 \mathrm{~ms}$ of response), having first confirmed that this yielded the same results as analyzing the whole response. 
A

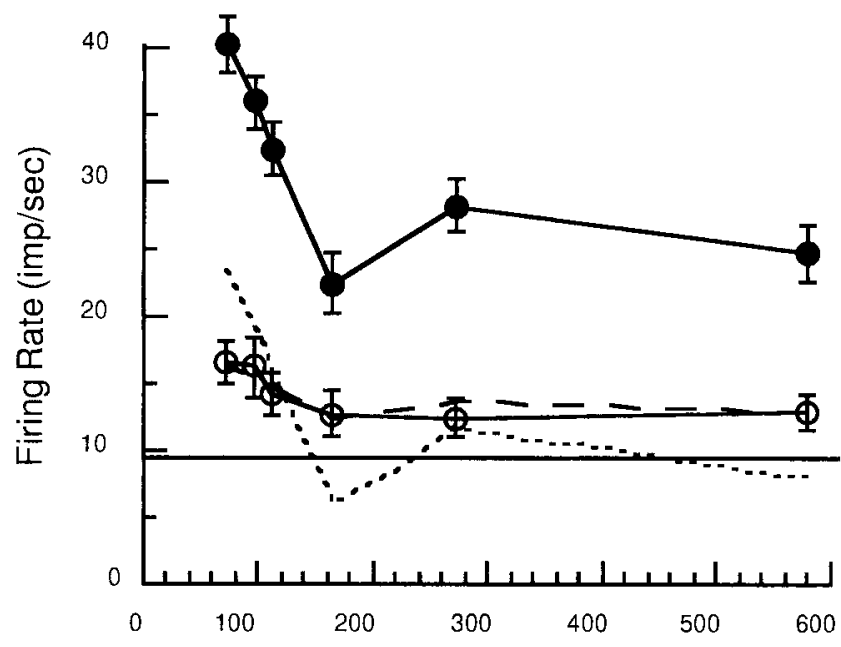

Time re onset of grating on receptive field (msec)
B

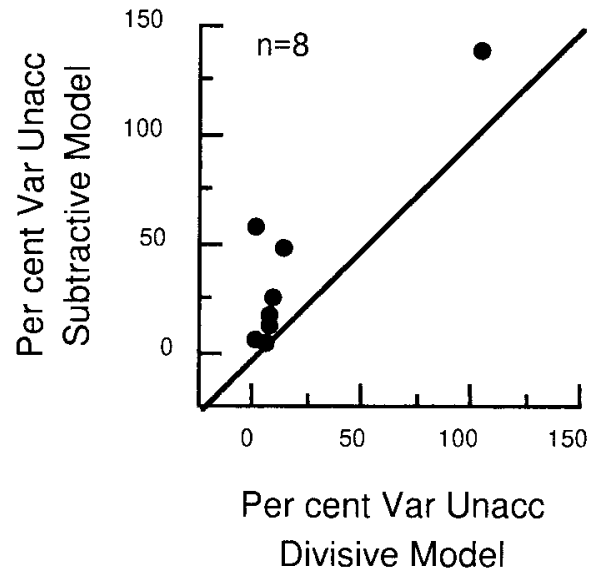

FIG. 6. A: response of a complex cell after the onset of a stationary grating confined to the receptive field, sampled in 100-ms epochs, each beginning at the poststimulus time indicated by the symbols (some of these epochs overlap), alone ( $A$ ), and in the presence of a surrounding grating presented just before one of the sampling epochs (by a fixed interval equal to the latency of response onset), so this added grating begins to influence discharge immediately before that epoch begins $(B)$. Error bars show \pm 1 SE. Horizontal rule indicates the maintained discharge. Dotted trace indicates the response expected if the surround acted subtractively. It shows the response to the receptive field stimulus alone, shifted down to provide the best fit to the response in the presence of the surrounding grating. Dashed trace shows the response expected if the surround acted divisively. This response is calculated as the response to the receptive field stimulus alone, scaled so as to best fit the response after addition of the surround. Expected responses are computed relative to the maintained discharge. $B$ : for each of 8 neurons, percentage of variance in the data unaccounted for by the best-fitting subtractive model vs. the best-fitting divisive model. Percentage of variance unaccounted for is the model's mean-squared fit error divided by the mean-squared error of the best-fit horizontal line.

Figure 7, $A$ and $B$, shows for two complex cells how the orientation of a surrounding grating influenced orientation selectivity measured with a grating confined to the receptive field. By depressing responsivity to gratings in the neighborhood of its own orientation and (for the neuron in Fig. $7 B$ ) increasing responsivity to gratings in other orientations, the surrounding grating repels the peak of the tuning curve. The surrounding grating also reduces the variability of the response to neighboring orientations (e.g., error bars in Fig. $7 B$ ). Figure $7 C$ shows, for a simple cell, that orientation selectivity is not systematically affected by the orientation of a surrounding grating. Responses are suppressed, and orientation selectivity is even changed but without regard for the orientation of the surrounding grating.

To quantify the deformation in orientation tuning illustrated in Fig. 7, we computed the center-of-mass $\sum i R_{i} / \sum R_{i}$ (see METHODS) for all the cells on which we made measurements. Figure 8 shows, for 32 complex cells and 9 simple cells, the center of mass found in the presence of a surrounding grating inclined to one side of the preferred orientation (usually by $14^{\circ}$ ) against the center of mass found in the presence of a grating inclined by the same amount to the other side of the preferred orientation. For complex cells ( and ) the surrounding grating often repels (and never attracts) the center of mass. Exchanging one surrounding grating for the other moves the center of mass by $4^{\circ}$ on average. For simple cells $(\bigcirc)$ a surrounding grating often reduced responsivity, but in $8 / 9$ neurons we studied, orientation tuning was not influenced by the orientation of the surrounding grating. Thus in Fig. 8, 8/9 simple cells fall along the unit diagonal.

A surrounding grating selectively depresses the responsivity of a complex cell to a central grating of similar orientation, making the tuning curve locally steeper and less variable from trial to trial. Might it thus improve the neuron's capacity to distinguish gratings of orientations near the orientation of the surround? We examined this by estimating the discriminability of two gratings, identical except for a $14^{\circ}$ (or $36^{\circ}$ ) difference in orientation. We measured a series of responses to each, and for the resulting distributions of spike counts calculated $d^{\prime}$, the difference between the mean counts, divided by the SD. When the two distributions had different SDs, we used the root-meansquare $\mathrm{SD}$, thus

$$
d^{\prime}=\frac{\mu_{1}-\mu_{2}}{\sqrt{\left(\sigma_{1}^{2}+\sigma_{2}^{2}\right) / 2}}
$$

(Green and Swets 1966). We measured responses twice, once with and once without, a surrounding grating whose orientation matched one of the test gratings. Among the 32 neurons for which we made this comparison the presence of the surrounding grating increased $d^{\prime}$ significantly $(t$-value increased by $\geq 2)$ in 9 but decreased it significantly in 5. A surrounding pattern that depresses responsivity thus appears to have no systematic effect on the discriminability of patterns falling in the receptive field.

\section{I S C U S S I O N}

\section{Influence on redundancy of representation}

By depressing responsivity and changing a complex cell's tuning locally in stimulus space, a surrounding grating will necessarily reduce correlation among the discharge rates (as distinct from the often-discussed correlation in spike times) of the population of neurons that respond to a particular stimulus (e.g., a grating of a particular orientation). This will increase 
A

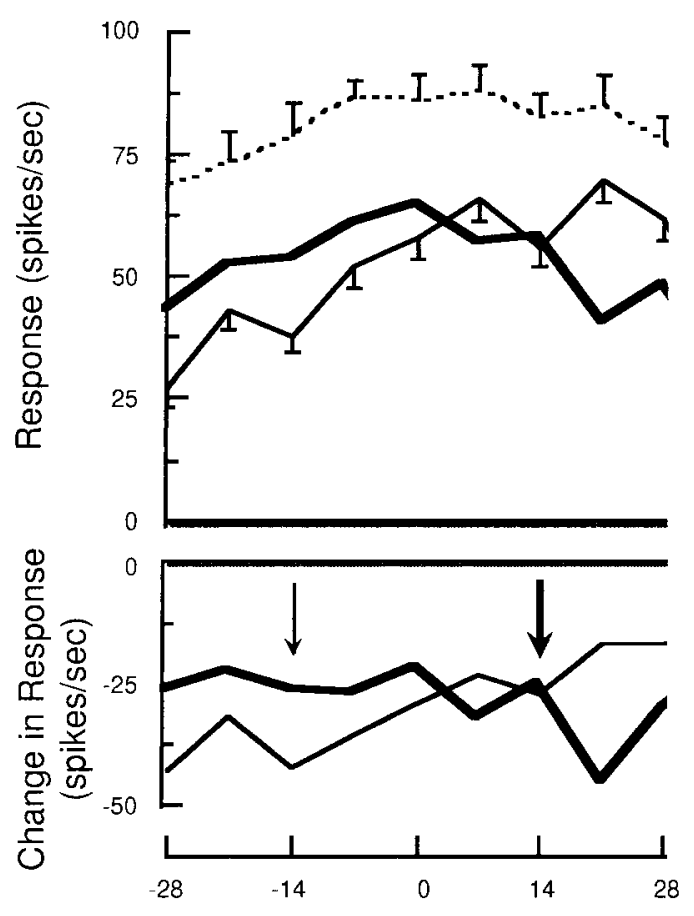

C

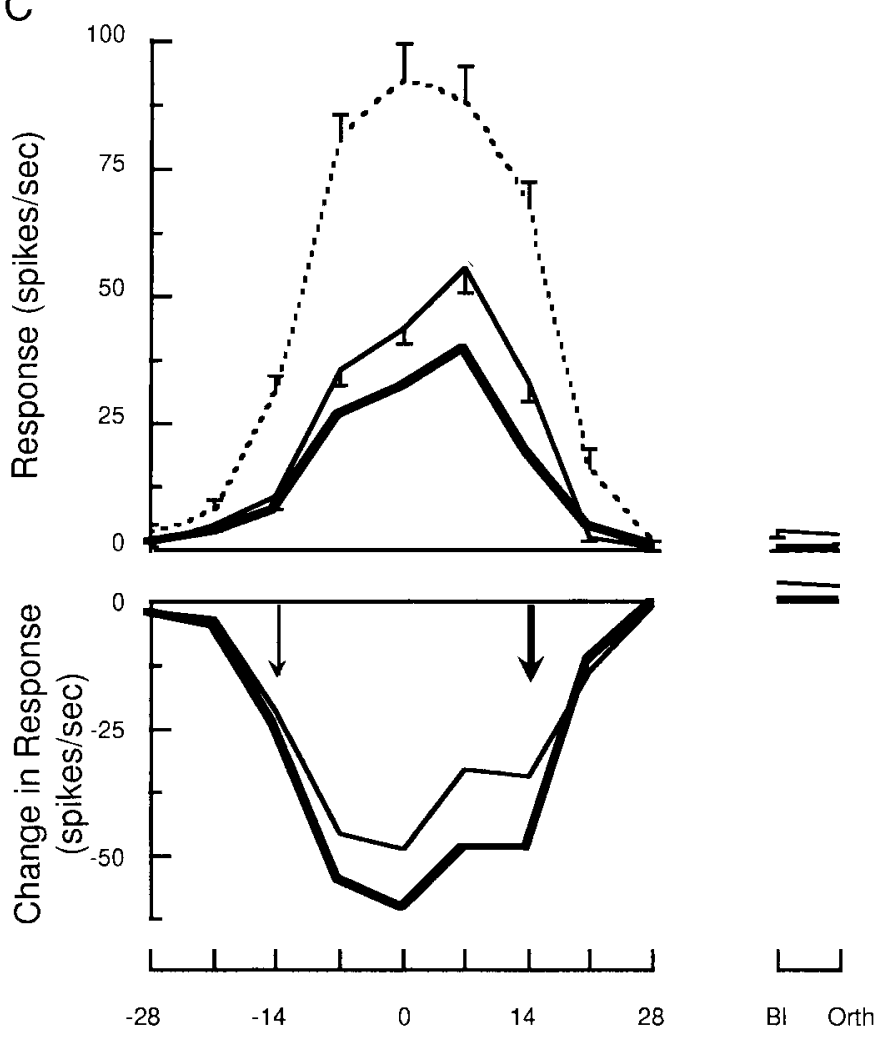

\section{B}

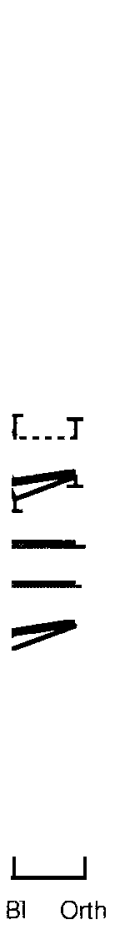

(20)

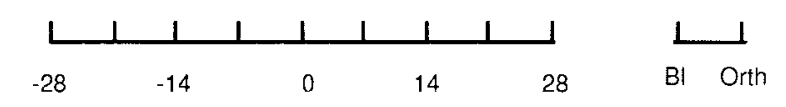

Orientation of central grating re preferred

Orientation of central grating re preferred 


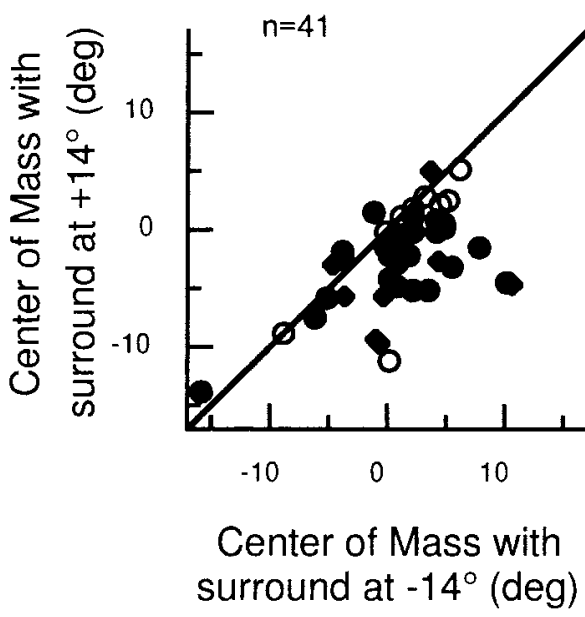

FIG. 8. Change in the orientation tuning of neurons brought about by surrounding gratings inclined to the neurons' preferred orientations. For each of 32 complex cells ( and $\bullet$ ) and 9 simple cells (O) the center of mass of the orientation tuning curve obtained in the presence of a surrounding grating tilted $14^{\circ}\left(\bullet\right.$ and $\bigcirc$ ) or $36^{\circ}(\bullet)$ from the preferred orientation (computed re the center of mass obtained when a single grating is presented alone) is plotted against the center of mass of the curve obtained with a counterpart grating inclined to the other side of the preferred orientation (computed in the same way). For explanation of the center-of-mass calculation, see METHODS.

the information transmitted by each spike (Barlow and Földiák 1989). Consider how the responsiveness of a population of neurons that have neighboring or overlapping receptive fields and are tuned to similar orientations will be changed by a single grating that surrounds all their receptive fields. We simulate such a population by taking the neurons from Fig. 8 (32 complex cells, 9 simple cells, studied with 2 surround orientations) and normalizing their actual tuning curves so that the surround has an orientation of $0^{\circ}$. This results in a simulated population of neurons most with preferred orientation $-14,0$, or $14^{\circ}$. Figure $9 \mathrm{~A}$ shows orientation tuning of two complex cells, each measured with a grating confined to its receptive field, presented alone (dotted traces) and with a surrounding grating at nominal orientation $0^{\circ}$ (solid traces with symbols). The surrounding grating sharply reduced each complex cell's response to gratings with similar orientations, and pushed apart the neurons' preferred orientations. Whenever a single grating extends across the receptive fields of a population of complex cells, these changes in tuning will make their responses less redundant.

To quantify the redundancy of the simulated population's response rates and the surround's role in reducing it, we compute the pairwise cross-correlation between the response rates of the population of neurons weighted according to the probability of a stimulus of each orientation. Thus we define redundancy $(\Psi)$ in the population's response to each grating of orientation $\theta$ falling in the receptive field as

$$
\Psi(\theta)=\sum_{\text {all cells }} \frac{\operatorname{Resp}(\operatorname{cell~A}, \theta) \cdot \operatorname{Resp}(\operatorname{cell~} \mathrm{B}, \theta)}{\text { number of cell pairs }}
$$

A
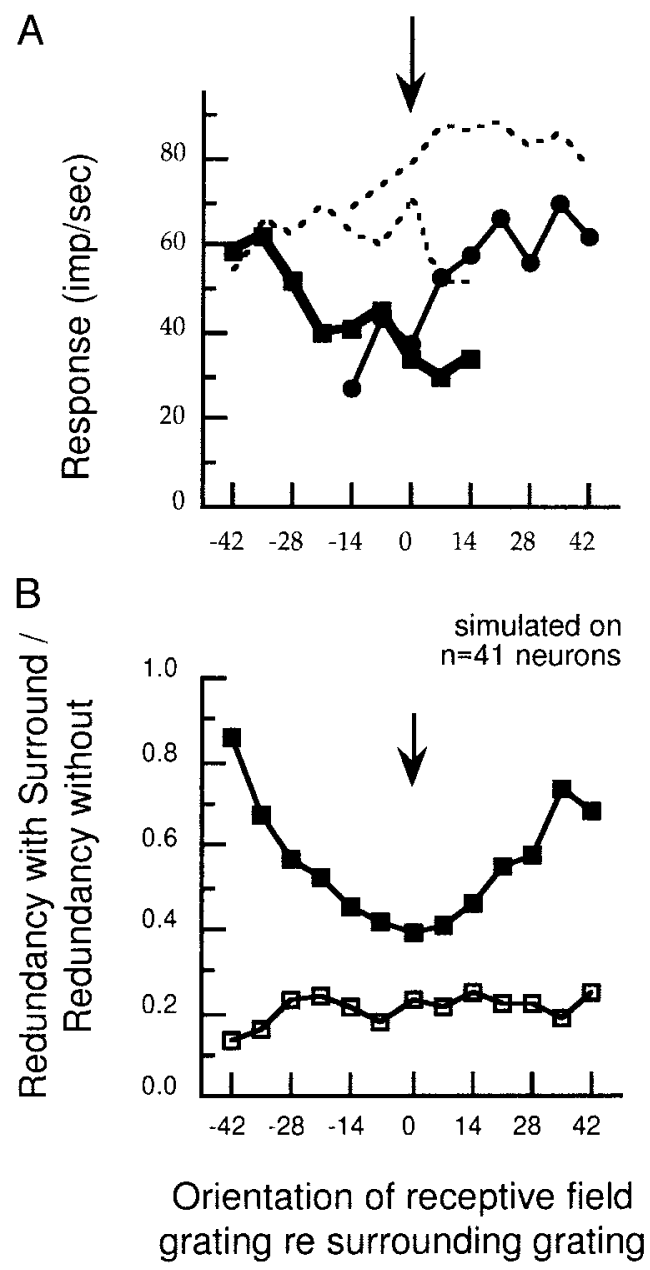

FIG. 9. Illustration of how a surrounding grating will reduce the redundancy (correlation) among responses of a population of neurons that all have preferred orientations near its orientation. $A$ : tuning curves for 2 complex cells measured in the absence (--) and presence ( - with filled symbols) of a surrounding grating at orientation $0^{\circ}(\rightarrow)$. B: average reduction brought about by a surround in the redundancy $\Psi(\theta)(E q .3)$ among responses of a group of

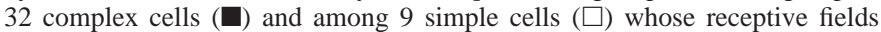
were stimulated by gratings at a range of orientations around the surrounding orientation.

the average, unnormalized, point-by-point product of the responses of all pairs of neurons. This is an intermediate stage in the calculation of the cross-correlation. If the response rates of the population of neurons were all statistically independent, then by definition this correlation measure would approach zero at each orientation.

The measure $\Psi$ can be used to show how surrounding gratings would reduce redundancy among the response rates of the simulated population. We calculate $\Psi$ with and without a surround for gratings presented to the receptive field at a range of orientations. Figure $9 B(\square)$ shows the average reduction in redundancy at each

FIG. 7. Influence of surrounding gratings of different orientations on the orientation tuning of 2 complex cells $(A$ and $B)$ and 1 simple cell $(C)$. Orientation tuning was measured from the 1 st $100 \mathrm{~ms}$ of response to high-contrast gratings of optimal spatial frequency and size, either stationary at the optimal spatial phase $(A$ and $C)$ or moving at the optimal temporal frequency, initially at the optimal spatial phase $(B)$. Different curves show tuning for the test grating presented alone (- - ) and in the presence of a surrounding grating lying at $-14^{\circ}(-)$ or $+14^{\circ}(-)$ from the preferred orientation $\left(0^{\circ}\right)$. Rightmost positions on the abscissas indicate that the test grating is replaced with a blank or is orthogonal to that preferred by the neuron. Thin and thick arrows mark the orientations of surrounding gratings. Error bars, where present, show \pm 1 SE. Bottom: the change in response to the test grating brought about by the surrounding grating. 
orientation (relative to the orientation of the surrounding grating) for pairs of neurons drawn from our population of 32 complex cells. Naturally, redundancy is most reduced when firing rate is most reduced: when the orientation of the grating falling on the receptive field is near that of the surround. The orientationdependent reduction in redundancy arises because the surrounding grating changes the orientation selectivities of neurons. This happens without reducing the discriminability of patterns falling on the receptive field (see RESULTS). The redundancy among the responses of our population of nine simple cells (open squares) was also reduced by the surround but to a uniform value at every orientation. This is because, in simple cells, surround stimulation scales down the entire orientation tuning curve without systematically changing its shape (Fig. 7C).

The reduced redundancy among the responses of the population of neurons tuned to a particular orientation will increase the information transmitted by each spike (Barlow 1990) and saving energy (Lennie 2003). Benefits will be greatest when parts of the image falling just outside the receptive field (on the surround) have nearly the same structure as the parts falling on the receptive field: the more a pattern falling on the receptive field resembles those that surround it, the greater the reduction in redundancy among the responses of the population. This behavior seems well matched to the properties of natural scenes: Simoncelli and Schwartz (1999) have shown, for a small sample of natural images, that statistics of iso-oriented filters responding to adjacent image regions are highly correlated, whereas those from cross-oriented filters or more separated regions are less well correlated. This encourages us to think of inhibitory lateral interaction as a phenomenon that complements the rapid adaptation described by Müller et al. (1999): both remove local correlations from neuronal signals, one in time, the other in space.

\section{Relation to other findings}

Gilbert and Wiesel (1990) and Sengpiel et al. (1997) reported (sometimes large) changes in preferred orientations of neurons in cat cortex brought about by surrounding gratings but did not find the consistent repulsion of preferred orientation that we observed here in complex cells.

Knierim and Van Essen's 1992 observations on latencies of surround influences in awake monkey (Table 1) are consistent with our finding that surrounds begin to suppress discharge as soon as neurons begin responding, though Knierim and Van Essen found significant suppression only after a delay. Zipser et al. (1996) studied how V1 neurons in awake monkeys responded to patterns that covered and extended beyond the receptive field and were embedded in a larger surrounding pattern that had the same or contrasting structure. The influence of such surrounding patterns is expressed 80-100 ms after the beginning of the response to the central pattern. Moreover, it influences responses only in the awake monkey (Lamme et al. 1998). Because of this, and the fact that even the central patterns used by Zipser et al. would have stimulated the kind of surround we have characterized, we think this long-latency phenomenon is unrelated to the one studied here. In anesthetized monkeys, Bair et al. (1999) stimulated receptive field and surround independently with a continuous series of very briefly presented gratings in four possible pairings of preferred and orthogonal orientations and found that the surround's suppressive effect was delayed 20-30 ms. The temporal structure of stimula- tion - an uninterrupted train of high-contrast stimuli-was quite unlike that provided by our discrete trials with intervening blanks and probably results in neurons having lower contrast gain. How that influences surround latency remains to be determined.

\section{Origin of surround signals}

Two broad classes of accounts have been offered of the origin of surround signals: they are conveyed via lateral connections within V1, such as those described by Rockland and Lund (1983) and Gilbert and Wiesel (1983) or they are conveyed through feedback connections from extrastriate cortex, an idea suggested by the observation that some surround effects are expressed with long latencies (Zipser et al. 1996).

In normally functioning visual cortex, synaptic delays are probably $\geq 5$ ms (Maunsell and Gibson 1992). Two or more of these, amounting to $\geq 10 \mathrm{~ms}$, would be required for a feedback signal from extrastriate cortex to influence a V1 neuron. If such a feedback loop originated in V1 neurons of the kind we have studied here, signal transmission time would be too long to explain the often-instantaneous action of the surround. Could a feedback loop originate in V1 in signals that we have not characterized? One possible source might be a class of shortlatency relay neurons that itself does not express any immediate surround influence and that we have not studied. We think it unlikely that such neurons exist. Our method for measuring latency (Fig. 3) can identify the earliest times at which signals are present in V1 neurons (and therefore available to any feedback loop). The shortest surround latencies match the shortest latencies for stimuli in the receptive field and thus too short to be plausibly the result of feedback (Fig. 4B).

The short latency of surround signals, their circumscribed range (see following text), and their asymmetrical/irregular weight around the receptive field (Cavanaugh et al. 2002b; Walker et al. 1999) make it unlikely that they arise in feedback from extrastriate cortex. Might they arise in connections from neurons within V1 (Das and Gilbert 1999)? In the region of V1 from which we made our recordings strong surround signals, which most often originate within $0.5^{\circ}$ of the receptive field (Born and Tootell 1991; Maffei and Fiorentini 1976), will most often need to be propagated over $2 \mathrm{~mm}$ or less because the magnification factor is $\sim 4 \mathrm{~mm} /{ }^{\circ}$ (Van Essen et al. 1984) (Fig. 6). Some inhibitory interneurons have axonal arbors as much as $800 \mu \mathrm{m}$ across (Lund and Wu 1997) and so could carry signals from much or most of a neuron's surround. Signals from the LGN could drive interneurons to give rise to surround signals that appear to act as fast as those arising in the receptive field. Membrane depolarization that would normally lead to a spike begins $\sim 10 \mathrm{~ms}$ before the spike arises (Azouz and Gray 1999), so a surround signal (either hyperpolarizing or depolarizing) that arrived $\leq 10 \mathrm{~ms}$ later than the normal driving signal could alter the time at which the spiking threshold was reached. Although delayed, that surround signal would appear to act sooner than the normal drive to the receptive field. Presumed inhibitory interneurons in visual cortex studied in vivo have shorter visual latencies than presumed relay cells (Mancilla et al. 1998). All this can be explained by surround signals that arise not from feedback from extrastriate cortex, but in connections from neurons within V1. 


\section{Relation to other contrast gain controls}

Surround signals act divisively to regulate sensitivity (Fig. 9) (also Cavanaugh et al. 2002a) and are just one class of several in cortex that regulate contrast gain but do not themselves drive a cell. Cross-orientation inhibition (Bonds 1989; DeAngelis et al. 1992) and null-phase inhibition (Geisler and Albrecht 1992) within the receptive fields of simple cells act this way. The latter phenomena can be explained by models that assume a neuron's contrast sensitivity is regulated ("normalized") by a pooled signal from a large number of neurons with overlying receptive fields covering all orientations (Carandini et al. 1997; Heeger 1992). Because this pooled signal is isotropic, it probably does not give rise to the surround inhibition studied here.

Rapid contrast adaptation (Müller et al. 1999) is another phenomenon that regulates contrast gain via a mechanism that also seems to be unlike that used by the surround. Nevertheless, much like the surround it brings about pattern-selective changes in the orientation tuning of complex cells only and does so without impairing a complex cell's capacity to discriminate orientation. These shared characteristics can be explained by supposing that the fundamental gain-controlling operation, whether lateral influence from the surround or rapid adaptation, originates in simple cells and that several simple cells with a range of orientation preferences are the subunits that provide input to each complex cell.

Present addresses: J. R. Müller, Howard Hughes Medical Institute and Dept. of Neurobiology, Fairchild D209, Stanford University School of Medicine, Stanford, CA 94305-5125; A. B. Metha, Dept. of Optometry and Vision Sciences, University of Melbourne, Carlton, Victoria, 3053 Australia; and P. Lennie, Center for Neural Science, 4 Washington Place, Room 809, New York University, New York, NY 10003.

\section{I S C L OS URES}

This work was supported by National Eye Institute Grants EY-04440, EY-01319, EY-06638, and EY-07125.

\section{REFERENCES}

Azouz R and Gray CM. Cellular mechanisms contributing to response variability of cortical neurons in vivo. J Neurosci 19: 2209-2223, 1999.

Bair W, Cavanaugh JR, and Movshon JA. The time course of contextual modulation in macaque V1 neurons. Invest Ophthalmol Vis Sci 40: S201, 1999.

Barlow HB. A theory about the functional role and synaptic mechanisms of visual aftereffects. In: Vision: Coding and Efficiency, edited by Blakemore C. Cambridge, UK: Cambridge Univ. Press, 1990, p. 363-375.

Barlow HB and Földiák P. Adaptation and decorrelation in the cortex. In: The Computing Neuron, edited by Durbin RM, Miall C, and Michison GJ. New York: Addison-Wesley, 1989, p. 54-72.

Blakemore $\mathbf{C}$ and Tobin EA. Lateral inhibition between orientation detectors in the cat's visual cortex. Exp Brain Res 15: 439-440, 1972.

Bonds AB. Role of inhibition in the specification of orientation-selectivity of cells in the cat striate cortex. Vis Neurosci 2: 41-55, 1989.

Born RT and Tootell RBH. Single-unit and 2-deoxyglucose studies of side inhibition in macaque striate cortex. Proc Natl Acad Sci USA 88: 7071-7075, 1991.

Bradley DC and Andersen RA. Center-surround antagonism based on disparity in primate area MT. J Neurosci 18: 7552-7565, 1998.

Carandini M, Heeger DJ, and Movshon JA. Linearity and normalization in simple cells of the macaque primary visual cortex. J Neurosci 17: 8621-8644, 1997.

Cavanaugh JR, Bair W, and Movshon JA. Nature and interaction of signals from the receptive field center and surround in macaque V1 neurons. J Neurophysiol 88: 2530-2546, 2002a.

Cavanaugh JR, Bair W, and Movshon JA. Selectivity and spatial distribution of signals from the receptive field surround in macaque v1 neurons. J Neurophysiol 88: 2547-2556, 2002b.
Das A and Gilbert CD. Topography of contextual modulations mediated by short-range interactions in primary visual cortex. Nature 399: 655-661, 1999.

DeAngelis GC, Freeman RD, and Ohzawa I. Length and width tuning of neurons in the cat's primary visual cortex. J Neurophysiol 71: 347-374, 1994.

DeAngelis GC, Robson JG, Ohzawa I, and Freeman RD. Organization of suppression in receptive fields of neurons in cat visual cortex. $J$ Neurophysiol 68: 144-163, 1992.

Edgington ES. Randomization Tests. New York: Dekker, 1995.

Forte J, Peirce JW, Kraft JM, Krauskopf J, and Lennie P. Residual eye-movements in macaque and their effects on visual responses of neurons. Vis Neurosci 19: 31-38, 2002.

Geisler WS and Albrecht DG. Cortical neurons: isolation of contrast gain control. Vision Res 32: 1409-1410, 1992.

Gilbert CD and Wiesel TN. Clustered intrinsic connections in cat visual cortex. J Neurosci 3: 1116-1133, 1983.

Gilbert CD and Wiesel TN. The influence of contextual stimuli on the orientational selectivity of cells in primary visual cortex of the cat. Vision Res 30: 1689-1701, 1990.

Green DM and Swets JA. Signal Detection Theory and Psychophysics. New York: Wiley, 1966.

Heeger DJ. Normalization of cell responses in cat striate cortex. Vis Neurosci 9: 181-197, 1992.

Hubel DH and Wiesel TN. Binocular interaction in striate cortex of kittens reared with artificial squint. J Neurophysiol 28: 1040-1059, 1965.

Knierim JJ and Van Essen DC. Neuronal responses to static texture patterns in area V1 of the alert macaque monkey. J Neurophysiol 67: 961-980, 1992.

Lamme VAF. The neurophysiology of figure-ground segregation in primary visual cortex. J Neurosci 15: 1605-1615, 1995.

Lamme VAF, Zipser K, and Spekreijse H. Figure-ground activity in primary visual cortex is suppressed by anesthesia. Proc Natl Acad Sci USA 95: 3263-3268, 1998.

Lennie P. The cost of cortical computation. Curr Biol 13: 493-497, 2003.

Levitt JB and Lund JS. Contrast dependence of contextual effects in primate visual cortex. Nature 387: 73-76, 1997.

Levitt JB and Lund JS. The spatial extent over which neurons in macaque striate cortex pool visual signals. Vis Neurosci 19: 439-452, 2002.

Lund JS and Wu CQ. Local circuit neurons of macaque monkey striate cortex. IV. Neurons of laminae 1-3A. J Comp Neurol 384: 109-126, 1997.

Maffei L and Fiorentini A. The unresponsive regions of visual cortical receptive fields. Vision Res 16: 1131-1139, 1976.

Mancilla JG, Fowler M, and Ulinski PS. Responses of regular spiking and fast spiking cells in turtle visual cortex to light flashes. Vis Neurosci 15: 979-993, 1998.

Maunsell JRH and Gibson JR. Visual response latencies in striate cortex of the macaque monkey. J Neurophysiol 68: 1332-1344, 1992.

Müller JR, Metha AB, Krauskopf J, and Lennie P. Information conveyed by onset transients in responses of striate cortical neurons. J Neurosci 21: 6978-6990, 2001.

Müller JR, Metha AB, Krauskopf J, and Lennie P. Rapid adaptation in visual cortex to the structure of images. Science 285: 1405-1408, 1999.

Nelson JI and Frost BJ. Orientation-selective inhibition from beyond the classical receptive field. Brain Res 139: 359-365, 1978

Rockland KS and Lund JS. Intrinsic laminar lattice connections in primate visual cortex. J Comp Neurol 216: 303-318, 1983.

Sengpiel F, Sen A, and Blakemore C. Characteristics of surround inhibition in cat area 17. Exp Brain Res 116: 216-228, 1997.

Sillito AM, Grieve KL, Jones HE, Cudeiro J, and Davis J. Visual cortical mechanisms detecting focal orientation discontinuities. Nature 378: 492-496, 1995.

Simoncelli EP and Schwartz O. Image statistics and cortical normalization models. In: Advances in Neural Information Processing Systems, edited by Kearns MS, Solla SA, and Cohn DA. Cambridge, MA: MIT Press, 1999.

Tanaka K, Hikosaka K, Saito H, Yukie M, Fukada Y, and Iwai E. Analysis of local and wide-field movements in the superior temporal visual areas of the macaque monkey. J Neurosci 6: 134-144, 1986.

Van Essen DC, Newsome WT, and Maunsell JHR. The visual field representation in striate cortex of the macaque monkey: asymmetries, anisotropies, and individual variability. Vision Res 24: 429-448, 1984.

Walker GA, Ohzawa I, and Freeman RD. Asymmetric suppression outside the classical receptive field of the visual cortex. J Neurosci 19: 10536-10553, 1999.

Zipser K, Lamme VAF, and Schiller PH. Contextual modulation in primary visual cortex. J Neurosci 16: 7376-7389, 1996. 